https://doi.org/10.22319/rmcp.v12i4.5796

Artículo

\title{
Relaciones entre estacionalidad, características corporales y leptina en el inicio de la pubertad en vaquillas Bos taurus taurus y Bos taurus indicus en el trópico mexicano
}

Carlos Hernández-López ${ }^{\text {a }}$

René Carlos Calderón-Robles ${ }^{b}$

Alejandro Villa-Godoy ${ }^{c}$

Ángel Ríos-Utrera ${ }^{\mathrm{d}}$

Sergio Iván Román-Ponce ${ }^{\mathrm{e}}$

Everardo González-Padilla ${ }^{\text {c* }}$

${ }^{a}$ Instituto Nacional de Investigaciones Forestales, Agrícolas y Pecuarias (INIFAP). Centro Nacional de Investigación Disciplinaria en Fisiología y Mejoramiento Animal. Querétaro, México.

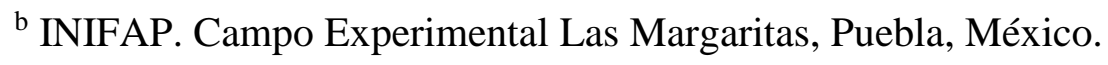

${ }^{\mathrm{c}}$ Universidad Nacional Autónoma de México. Facultad de Medicina Veterinaria y Zootecnia. Ciudad de México, México.

d INIFAP. Campo Experimental La Posta. Veracruz, México.

e INIFAP. Campo Experimental La Campana. Chihuahua, México.

* Autor de correspondencia: ever@unam.mx

\section{Resumen:}

El estudio analizó, en dos años, los efectos de raza [Brahman (BHM; n=65); Suizo Europeo $(\mathrm{SE} ; \mathrm{n}=56)$ ], suplementación con Zilpaterol@ (ZIL; tratadas o testigo), estación de nacimiento (primavera u otoño) y sus interacciones, sobre superficie corporal (SC), edad 
(EPB), peso corporal (PPB), condición corporal (CC), profundidad del músculo largo dorsal (MD), grosor de grasa dorsal (GD) y concentración sérica de leptina (LEP) a la pubertad (PB) de 121 vaquillas. A la $\mathrm{PB}$, las BHM fueron más pesadas y tuvieron más edad que las SE ( $376.8 \pm 7.4$ vs $302.0 \pm 6.6 \mathrm{~kg} ; 588.1 \pm 14.7$ vs $445.5 \pm 12.5$ días). ZIL aumentó EPB, PPB, CC y MD, pero no afectó GD y LEP. Las BHM tuvieron $18 \%$ más SC que las SE. Sin embargo, la diferencia en PPB/SC fue sólo de $6.4 \%$. Cuando se usó peso metabólico (PM) como proporción de la SC (PM/SC) en lugar de $\mathrm{PPB}$, la diferencia entre BHM y SE desapareció $(P>0.05)$. El GD fue $63.7 \%$ mayor en BHM que en SE. Las nacidas en primavera iniciaron la $\mathrm{PB}$ con $24.4 \%$ menos GD que las nacidas en otoño. La mayoría de las vaquillas BHM $(73.8 \%)$ inició la PB en los meses en que las horas luz iban en aumento $(P<0.05)$, mientras que en las SE el inicio de la PB estuvo uniformemente distribuido a través del año, independientemente de la duración de las horas luz; este efecto se mostró en los dos años de estudio. Se concluye que el establecimiento de la pubertad es un fenómeno multifactorial; la estacionalidad afecta a BHM y SE de manera diferenciada y, aparentemente, la SC es un factor importante, probablemente asociado con la eficiencia en la utilización de energía. Este trabajo reitera la importancia de la grasa dorsal y documenta, por primera vez PM/SC y su asociación con el establecimiento de la pubertad.

Palabras clave: Pubertad, Superficie corporal, Estacionalidad, Ganado tropical, Leptina.

Recibido: 04/09/2020

Aceptado: 03/09/2021

\section{Introducción}

Entre las determinantes más importantes para el establecimiento de la pubertad en vaquillas para producción de carne se incluyen la edad ${ }^{(1)}$, la talla y el peso corporal $^{(2)}$, con diferencias marcadas entre razas. En condiciones tropicales, actualmente son comunes los sistemas de producción con bovinos Bos taurus taurus, Bos taurus indicus y sus cruzas y del desempeño reproductivo de los hatos de cría depende la producción y productividad global del sistema de producción de carne de res. La etapa prepuberal de las vaquillas es un factor de costo y la edad a primer parto es un indicador importante del desempeño reproductivo del hato. Las vaquillas Cebú (Bos taurus indicus) requieren más edad y peso para alcanzar la pubertad que las Bos taurus taurus ${ }^{(3)}$. Este hecho se ha destacado como un factor limitante para el éxito reproductivo de las razas Bos taurus indicus ${ }^{(4)}$. La edad a la pubertad está estrechamente relacionada con el peso y la composición corporal de los animales ${ }^{(5)}$. 
Existen interacciones complejas entre diferentes hormonas para el establecimiento de la pubertad $^{(6)}$ y se resalta la importancia de la leptina por su asociación con la acumulación de grasa, ya que participa en la regulación del consumo de alimento, y es buen indicador dinámico de la condición corporal y del estado nutricional en rumiantes ${ }^{(7)}$. Se ha reportado que las concentraciones circulantes de leptina aumentan durante el desarrollo puberal en vaquillas $^{(8)}$. La información disponible sugiere que hay un nivel crítico de grasa requerido para el inicio de la actividad reproductiva ${ }^{(9)}$ y que puede haber diferencias en este límite crítico de grasa entre razas ${ }^{(10)}$, lo que implica que deben existir condiciones para regular la acumulación de energía excedente en forma de grasa corporal. A pesar de que se conocen varios factores que afectan el establecimiento de la pubertad en vaquillas, es escasa la literatura sobre las interacciones entre estos y, particularmente, sobre las diferencias entre $B$. taurus taurus y B. taurus indicus, en especial si consideramos el factor estacionalidad, que se asocia con el ganado Cebú( ${ }^{(3,11,12)}$. Con base en lo anterior, el presente estudio tuvo como objetivo determinar la asociación entre algunos señalizadores de la composición corporal, del ambiente interno de los animales y los del medio ambiente externo, con el establecimiento de la pubertad en vaquillas $B$. taurus taurus y B. taurus indicus, alimentadas en forma individual para tener ganancias de peso similares, nacidas en diferentes estaciones del año, con una composición corporal diferente, inducida durante su crecimiento.

\section{Material y métodos}

\section{Localización}

El estudio se realizó en el campo experimental Las Margaritas, dependiente del INIFAP, ubicado en Hueytamalco, Puebla, México, a 19 51' 03" LN y 97 12' 48" LO, a 500 msnm. El clima es subtropical húmedo semicálido $\mathrm{Af}(\mathrm{c})$, con temperatura promedio anual de 20.8 ${ }^{\circ} \mathrm{C}$, precipitación pluvial media anual de 3,000 mm y humedad relativa de $90 \%$.

\section{Tratamientos}

El estudio se repitió durante dos años; en total se utilizaron 121 vaquillas provenientes de dos estaciones de nacimiento: 24 Suizo Europeo (SE) y 33 Brahman (BHM) nacidas en primavera (04 de mayo \pm 36 días), y 32 SE y 32 BHM nacidas en otoño (27 de octubre \pm 35 días). La mitad de las vaquillas de cada raza y estación-año recibió uno de dos tratamientos: 1) con el $\beta$-agonista clorhidrato de Zilpaterol (ZIL, Zilpaterol®); $0.15 \mathrm{mg} / \mathrm{kg}$ de los 220 a los $300 \mathrm{~kg}$ de peso corporal y $0.25 \mathrm{mg} / \mathrm{kg}$ de $\operatorname{los} 301 \mathrm{~kg}$ de peso corporal hasta la primera ovulación, mezclado en el concentrado, y 2) $\sin \beta$-agonista (testigo). La PB para este estudio se definió como la primera ovulación que precedió al primer ciclo estral con formación de un cuerpo lúteo de duración normal ( $\geq 12 \leq 17$ días), correspondiente a un ciclo estral regular $(21 \pm 4 \text { días })^{(13)}$. 


\section{Manejo general}

Las vaquillas ingresaron al estudio con alrededor de siete meses de edad. Se alojaron de manera individual en corrales de $4 \times 6 \mathrm{~m}$, con piso de cemento, techo de asbesto $(4 \times 3 \mathrm{~m})$, comedero y bebedero. Para su adaptación al manejo y a la rutina de muestreo, las vaquillas se introdujeron a los corrales aproximadamente 30 días antes del estudio, se sujetaron con jáquima ( $2 \mathrm{~h}$ /día) y se cepillaron manualmente. La alimentación consistió en forraje (Saccharum sinense) fresco, picado, a libertad, y concentrado comercial (18\% de proteína cruda y $70 \%$ de TDN), cuya cantidad se ajustaba luego de cada pesaje de las vaquillas, para obtener ganancias de peso similares entre animales. Por cuestiones prácticas, se decidió nombrar de forma indistinta «vaquillas» a todas las hembras desde el inicio hasta su salida del experimento.

\section{Estimación de parámetros de desarrollo y composición corporal}

Los animales se pesaron cada 14 días, previo retiro de alimento y agua por 18 h. Cada 21 días se registró la condición corporal ( 1 a 9; $1=$ muy delgada, $9=$ obesa $)^{(14)}$, la cual fue calificada de manera independiente por tres personas, y el promedio de estas calificaciones se usó como variable de respuesta. La superficie corporal se midió cada 48 días, con un contador adaptado a un instrumento de rodillos para medir la superficie del tronco (odómetro); además, se empleó una cinta métrica para determinar la superficie de miembros, cabeza, orejas y cola. Para garantizar la reproducibilidad de las mediciones, el odómetro se validó mediante la medición independiente de 10 animales, por tres personas en tres ocasiones un mismo día. Se obtuvo un coeficiente de correlación intraclase de las medidas individuales y del promedio de 0.96 y 0.98 ( $P<0.0001)$, respectivamente, y una fiabilidad de 0.98 en el Alfa de Cronbach ${ }^{(15)}$. El área medida con el odómetro se estableció con la circunferencia de las llantas, la distancia entre las mismas y el número de vueltas.

Para calcular la superficie corporal (SC), se utilizó la siguiente fórmula: $\mathrm{SC}=2(a+p+m+$ $o)+r+c$, donde: $a=$ área medida con el odómetro, $p=$ área de la pierna, $m=$ área del miembro anterior, $o=$ área de la oreja, $r=$ área de la cola y $c=$ área de la cabeza. Las mediciones generales, hechas con cinta métrica, se ingresaron a una base de datos Excel y se validaron de la misma forma que el odómetro, obteniendo un coeficiente de correlación intraclase de 0.99 para las medidas individuales y el promedio $(P<0.0001)$. Se midió el grosor de la grasa dorsal (GD) y la profundidad del músculo largo dorsal (MD), obteniendo imágenes con un ultrasonido (equipado con un transductor de $3.5 \mathrm{MHz}$ ) en el lado izquierdo del dorso, a 12 $\mathrm{cm}$ de la línea media, a nivel de la doceava costilla, previa depilación del área y aplicación de gel $^{(16)}$. Las mediciones se hicieron cada 14 días, coincidiendo con los pesajes de los animales a lo largo del estudio. Las mediciones de MD corresponden solo a las vaquillas del segundo año de experimentación. 


\section{Obtención de sangre y medición de hormonas}

Al iniciar el estudio, para confirmar el estado prepuberal de las vaquillas mediante la progesterona sérica, se colectó una muestra de sangre de cada animal durante cinco días consecutivos, mediante punción de la vena yugular con aguja y tubos al vacío sin anticoagulante. Posteriormente, se colectaron muestras cuatro veces por semana hasta que cada vaquilla alcanzó $230 \mathrm{~kg}$ de peso corporal. A partir de ese momento el muestreo fue diario hasta finalizar el estudio, que fue cuando se determinó el inicio de la pubertad (PB), confirmado con la identificación de la primera ovulación mediante ultrasonido. Las muestras se procesaron para obtención de suero, el cual se congeló $-20^{\circ} \mathrm{C}$ hasta determinar por radioinmunoanálisis (RIA) la concentración de progesterona (P4). La concentración de $\mathrm{P} 4$ se utilizó como confirmación del estado prepuberal (valores $<1 \mathrm{ng} / \mathrm{ml}$ ) de las vaquillas. La concentración sérica de leptina (LEP) se evaluó con RIA específico para rumiantes ${ }^{(17)}$. Las concentraciones séricas de leptina se cuantificaron a partir de las muestras tomadas cuatro veces por semana desde el inicio del experimento. Una vez identificado el inicio de la PB, se seleccionaron las últimas 12 muestras previas a la primera ovulación, de las cuales se obtuvo el valor promedio de la concentración sérica de leptina a la pubertad; los datos obtenidos fueron únicamente de las vaquillas del segundo año de experimentación.

\section{Ultrasonografía de estructuras ováricas}

A partir de que se pudo introducir la mano por el recto de las vaquillas, aproximadamente a los $230 \mathrm{~kg}$ y 10 meses de edad, se tomaron imágenes ultrasonográficas de los ovarios para identificar la primera ovulación; inicialmente dos veces por semana y después diariamente. Para ello se utilizó un equipo Sonovet, con transductor rectal de $7.5 \mathrm{MHz}$ y videograbadora. La primera ovulación se consideró el primer día que se detectó tejido lúteo precedido por la desaparición súbita del folículo dominante, lo anterior corroborado con la P4 sérica.

\section{Variables}

Las variables de respuesta fueron los valores a la PB de: edad (EPB; días), peso corporal (PPB; $\mathrm{kg}$ ), peso metabólico (PM; peso corporal elevado a la 0.75 potencia; $\mathrm{kg}$ ), $\mathrm{SC}\left(\mathrm{m}^{2}\right)$, condición corporal (CC; puntos), $\mathrm{GD}(\mathrm{cm}), \mathrm{MD}(\mathrm{cm})$, peso corporal entre superficie corporal (PPB/SC; $\mathrm{g} / \mathrm{cm}^{2}$ ), peso metabólico entre superficie corporal (PM/SC; $\mathrm{g} / \mathrm{cm}^{2}$ ) y concentración sérica de leptina (LEP; ng/ml). Las variables PPB/SC y PM/SC se generaron para saber cuántos gramos de tejido metabólicamente activo hay por cada centímetro cuadrado de piel, que es un órgano que participa en la regulación de la temperatura corporal y puede actuar como un difusor de energía. 


\section{Análisis estadísticos}

Los datos se analizaron mediante análisis de varianza y correlación de Pearson. El diseño fue un completamente al azar con arreglo factorial $2 \times 2 \times 2$. El modelo estadístico preliminar incluyó los efectos principales de raza (RZ), estación de nacimiento (EN) y tratamiento (con o sin $\beta$-agonista), las interacciones dobles y triples entre estos efectos, el peso corporal de entrada al experimento como covariable y, como bloque, el grupo de entrada al experimento (animales agrupados según la fecha y año exacta de estudio) anidado en RZ x EN. El modelo estadístico definitivo incluyó los efectos principales y el bloque. Además, para EPB y CC, el modelo incluyó el peso corporal al inicio del experimento; la interacción RZ x EN solo se incluyó en el análisis definitivo de CC. Para analizar LEP, se realizó un análisis de covarianza de las concentraciones séricas de leptina, sobre los días transcurridos desde el inicio del estudio hasta el inicio de la PB, incluyendo los efectos fijos de RZ, EN y tratamiento. Las diferencias entre medias se determinaron con la opción PDIFF, todo esto con el procedimiento GLM de $\mathrm{SAS}^{(18)}$. Se realizó una prueba de homogeneidad con una Jicuadrada, para observar el efecto de estacionalidad a la PB de las vaquillas, utilizando las variables categóricas RZ y EN, con respecto a las horas luz (en aumento o disminución) a la PB.

\section{Resultados}

\section{Correlaciones entre variables}

Independientemente de la raza de las vaquillas, se encontró correlación alta entre PPB y EPB ( $\mathrm{r}=0.86 ; P<0.01)$, así como entre SC y EPB $(\mathrm{r}=0.84 ; P<0.01)$. Asimismo, la SC se correlacionó con PPB y PM $(\mathrm{r}=0.90 ; P<0.01)$. Para LEP y PPB se observó una baja correlación ( $\mathrm{r}=0.28 ; P<0.01)$, así como para LEP y SC $(\mathrm{r}=0.37 ; P<0.01)$. Se observó una correlación intermedia para las variables PM/SC y GD $(\mathrm{r}=0.52 ; P<0.01)$. La covariable peso de las vaquillas al inicio del experimento sólo resultó significativa para EPB $(P<0.01)$ y CC $(P<0.05)$. Se encontró efecto de la interacción RZ x EN sobre CC $(P<0.05)$. Además, hubo relación lineal $(P<0.01)$ de LEP con respecto a los días transcurridos hasta la $\mathrm{PB}$, existiendo $35 \mathrm{pg} / \mathrm{ml}$ más de LEP por cada día que se aproximó la pubertad.

\section{Efecto de raza sobre las variables relacionadas con la pubertad}

A la $\mathrm{PB}$, las vaquillas BHM fueron $74.8 \mathrm{~kg}$ más pesadas que las $\mathrm{SE}(P<0.0001)$, diferencia relacionada con la edad, ya que las BHM requirieron 142.6 d más que las SE para que la PB se presentara $\left(P<0.0001\right.$; Cuadro 1). Las vaquillas BHM tuvieron $0.64 \mathrm{~m}^{2}(18 \%)$ más de SC que las SE $(P<0.0001)$, mientras que la relación $\mathrm{PPB} / \mathrm{SC}$ fue $6.4 \%$ superior $(P<0.001)$ en las BHM; esta diferencia desapareció cuando la comparación se hizo con base en PM/SC. Las 
vaquillas BHM mostraron $0.4 \%$ más $(P<0.05)$ CC que las SE $(7.72$ vs 7.69 puntos $)$ a la PB. La diferencia más evidente fue en GD, donde las BHM mostraron $64 \%$ más GD a la PB que las SE $(P<0.0001)$. Coincidiendo con lo anterior, las vaquillas BHM tuvieron $20 \%$ más $(P<0.0001)$ LEP a la PB que las SE.

Cuadro 1: Medias de cuadrados mínimos y errores estándar para las variables de respuesta a la pubertad, para los efectos raza y tratamiento

\begin{tabular}{|c|c|c|c|c|c|}
\hline \multirow[b]{2}{*}{ Variable $^{1}$} & \multicolumn{2}{|l|}{ Raza } & \multicolumn{2}{|l|}{ Tratamiento } & \multirow[b]{2}{*}{ Promedio } \\
\hline & Brahman & \multicolumn{3}{|l|}{ Suizo } & \\
\hline $\mathrm{PPB}, \mathrm{kg}$ & 376.77 & 301.97 & 320.47 & $358.27 \pm$ & $339.37 \pm$ \\
\hline & $7.42^{\mathrm{a}}$ & $6.59^{b}$ & $6.25^{\mathrm{a}}$ & $6.42^{\mathrm{b}}$ & 6.78 \\
\hline $\mathrm{PM}, \mathrm{kg}$ & $85.34 \pm 1.29^{a}$ & $72.32 \pm 1.15^{\mathrm{b}}$ & $75.57 \pm 1.09^{\mathrm{a}}$ & $82.09 \pm 1.12^{b}$ & $78.83 \pm 1.18$ \\
\hline EPB, días & 588.13 & 445.51 & 490.29 & 543.35 & 516.82 \\
\hline & $14.71^{\mathrm{a}}$ & $12.48^{\mathrm{b}}$ & $11.96^{\mathrm{a}}$ & $12.42^{\mathrm{b}}$ & 13.08 \\
\hline $\mathrm{SC}, \mathrm{m}^{2}$ & $4.22 \pm 0.07^{\mathrm{a}}$ & $3.58 \pm 0.06^{b}$ & $3.79 \pm 0.05^{\mathrm{a}}$ & $4.00 \pm 0.06^{b}$ & $3.90 \pm 0.06$ \\
\hline $\begin{array}{l}\mathrm{PPB} / \mathrm{SC}, \\
\mathrm{g} / \mathrm{cm}^{2}\end{array}$ & $8.99 \pm 0.11^{\mathrm{a}}$ & $8.45 \pm 0.10^{\mathrm{b}}$ & $8.48 \pm 0.09^{\mathrm{a}}$ & $8.97 \pm 0.10^{\mathrm{b}}$ & $8.72 \pm 0.10$ \\
\hline $\mathrm{PM} / \mathrm{SC}, \mathrm{g} / \mathrm{cm}^{2}$ & $2.05 \pm 0.02^{\mathrm{a}}$ & $2.03 \pm 0.02^{\mathrm{a}}$ & $2.01 \pm 0.02^{\mathrm{a}}$ & $2.07 \pm 0.02^{\mathrm{b}}$ & $2.04 \pm 0.02$ \\
\hline $\mathrm{CC}, 1$ a 9 & $7.72 \pm 0.06^{\mathrm{a}}$ & $7.69 \pm 0.05^{b}$ & $7.56 \pm 0.05^{\mathrm{a}}$ & $7.86 \pm 0.05^{b}$ & $7.71 \pm 0.06$ \\
\hline $\mathrm{MD}, \mathrm{cm}^{*}$ & $5.86 \pm 0.10^{\mathrm{a}}$ & $4.69 \pm 0.10^{b}$ & $4.95 \pm 0.09^{\mathrm{a}}$ & $5.60 \pm 0.09^{b}$ & $5.28 \pm 0.10$ \\
\hline $\mathrm{GD}, \mathrm{cm}$ & $2.39 \pm 0.07^{\mathrm{a}}$ & $1.46 \pm 0.06^{\mathrm{b}}$ & $1.95 \pm 0.06^{\mathrm{a}}$ & $1.91 \pm 0.06^{\mathrm{a}}$ & $1.93 \pm 0.06$ \\
\hline $\mathrm{LEP}, \mathrm{ng} / \mathrm{ml} *$ & $3.30 \pm 0.10^{\mathrm{a}}$ & $2.75 \pm 0.09^{b}$ & $2.95 \pm 0.09^{\mathrm{a}}$ & $3.09 \pm 0.09^{\mathrm{a}}$ & $3.02 \pm 0.09$ \\
\hline
\end{tabular}

${ }^{1} \mathrm{PPB}=$ peso corporal; $\mathrm{PM}=$ peso metabólico; $\mathrm{EPB}=$ edad; $\mathrm{SC}=$ superficie corporal; $\mathrm{PPB} / \mathrm{SC}=$ peso corporal entre superficie corporal; $\mathrm{PM} / \mathrm{SC}=$ peso metabólico entre superficie corporal; $\mathrm{CC}=$ condición corporal; $\mathrm{MD}=$ profundidad del músculo largo dorsal; $\mathrm{GD}=$ grosor de la grasa dorsal; $\mathrm{LEP}=$ concentración sérica de leptina.

*Resultados del segundo año.

${ }^{a, b}$ Medias con distinta literal entre columnas de cada efecto fijo en cada una de las variables de respuesta indican diferencia $(P<0.05)$.

\section{Efecto del tratamiento sobre las variables relacionadas con el inicio de la pubertad}

El tratamiento con ZIL tuvo un efecto significativo $(P<0.01)$ sobre el PPB; los animales que recibieron ZIL requirieron $37.8 \mathrm{~kg}$ más de peso corporal para iniciar la PB. En cuanto a EPB, tardaron 53 días más que los no tratados $(P<0.05)$; además, su SC y $\mathrm{PPB} / \mathrm{SC}$ fueron 7.0 $(P<0.001)$ y $6.0 \%$ mayores $(P<0.05)$, respectivamente. La CC y la MD a la PB de los animales suplementados con ZIL fue 4.0 y $13.1 \%$ mayor $(P<0.001)$ que la de los del grupo control, respectivamente. Por el contrario, no se registró diferencia significativa $(P>0.05)$ entre los dos grupos con respecto a GD y LEP. 
Figura 1: Medias de cuadrados mínimos y errores estándar para condición corporal (CC) a la pubertad, por raza y estación de nacimiento

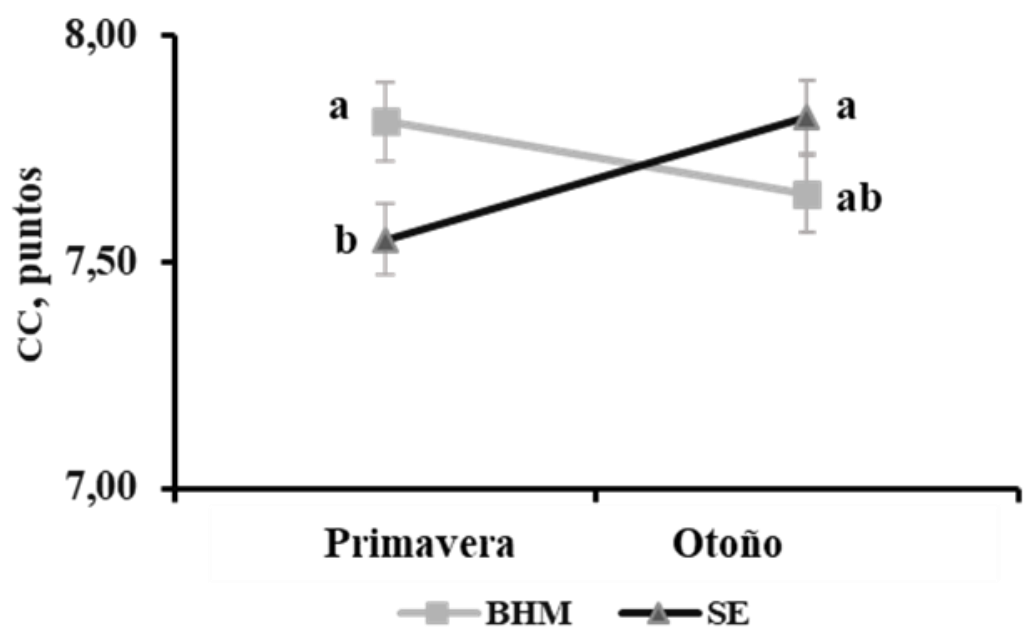

$\mathrm{BHM}=\mathrm{Brahman}, \mathrm{SE}=$ Suizo Europeo. Literales distintas $(\mathrm{a}, \mathrm{b})$ entre medias indican diferencia $(P<0.05)$.

Se observó que 48 de las 65 vaquillas BHM iniciaron la PB en los meses en los que las horas luz aumentaban ( 22 de diciembre al 21 de junio) y las otras 17 vaquillas lo hicieron en los meses en los que las horas luz disminuían (22 de junio al 21 de diciembre) $(P<0.05)$. Por el contrario, las vaquillas SE iniciaron la PB independientemente de la tendencia de cambio del fotoperiodo, ya que 30 de ellas iniciaron la PB cuando las horas luz aumentaban y otras 26 lo hicieron cuando las horas luz disminuían $(P>0.05)$.

\section{Discusión}

El presente estudio aporta información relevante sobre las relaciones entre señalizadores de la composición corporal, del ambiente interno de los animales y los del medio externo con el establecimiento de la pubertad en vaquillas. Se destaca la relación del establecimiento de la PB con características corporales como la cantidad de grasa y la SC; para esta última, la diferencia mostrada entre razas (17.8\%) se redujo al incorporar en el análisis PPB/SC (6.4\%), pero desapareció al considerarse $\mathrm{PM} / \mathrm{SC}(<1 \% ; P>0.05)$, lo que sugiere que más que la SC en sí, lo importante es la relación de la masa corporal con la SC, independientemente de la raza, a pesar de las claras y significativas $(P<0.05)$ diferencias a la $\mathrm{PB}$ en el resto de las variables estudiadas entre BHM y SE. En buena medida, las diferencias en varias características corporales entre BHM y SE a la PB pudieron estar asociadas al hecho de que la PB en BHM ocurrió con 4.6 meses más de edad, período en que siguieron creciendo y modificando su composición corporal, sin embargo, no existió diferencia en la relación $\mathrm{PM} / \mathrm{SC}$ a la $\mathrm{PB}$, lo que sugiere que éste es un parámetro importante; si no es detonante, al 
menos indicativo de que hay un balance de energía que permite cubrir la demanda de las funciones vitales, termorregulación, locomoción, crecimiento, y hay un excedente a destinar para procesos reproductivos ${ }^{(19)}$.

El tratamiento con zilpaterol a la mitad de los animales para inducir una composición corporal diferente entre estos y los del grupo control, permitió observar que a pesar de las diferencias a la PB en cuanto a las variables peso, edad y características corporales, debidas al tratamiento, GD y LEP no mostraron diferencias entre los grupos ZIL y testigo, lo que destaca la importancia de la composición corporal y, en particular, de la cantidad de grasa corporal para el establecimiento de la PB.

Se ha reportado que en el crecimiento y la composición de la canal, existe una gran variación entre B. taurus taurus ${ }^{(20,21)}$ y B. taurus indicus ${ }^{(22)}$. Así mismo, existen diferencias en la distribución de la grasa corporal entre razas; las razas lecheras depositan mayor proporción de su grasa internamente y menor proporción de forma subcutánea que las de carne ${ }^{(23)}$. Estas diferencias, independientemente de los factores ambientales, aparentemente participan en los procesos de maduración para que los individuos sean cronológicamente distintos entre razas $^{(24)}$, con la consecuente variación en la edad al primer parto, que es una variable de importancia económica. La edad estimada a la PB del ganado Cebú en los trópicos y subtrópicos oscila ampliamente entre 16 y 40 meses $^{(25)}$ y, en consecuencia, la edad al primer parto es igualmente muy variable debido al efecto que tienen variables ambientales, destacándose la alimentación de los animales, que en condiciones de pastoreo depende de condicionantes como la precipitación pluvial, la calidad y fertilidad de los suelos y las especies forrajeras en uso, particularmente en condiciones tropicales, de tal forma que el estudio de fenómenos fisiológicos, como el establecimiento de la pubertad y los efectos estacionales en procesos reproductivos, requieren del control de variables como la alimentación, que puede confundir los resultados. Por esa razón, para este trabajo se alimentó individualmente a los animales para que todos tuvieran una ganancia diaria similar. La ganancia diaria de peso promedio general fue de $560 \pm 121 \mathrm{~g} /$ día.

A la PB, las vaquillas BHM fueron más pesadas y tuvieron mayor edad que las SE; de hecho, esos 142 días de diferencia implicaron que BHM tuviera en promedio $74.8 \mathrm{~kg}$ más (en congruencia con la ganancia promedio de peso de las vaquillas durante el estudio), sin embargo, por la diferencia en SC no hubo diferencia en PM/SC entre las dos razas. Se observó una correlación de $0.96(P<0.01)$ entre PPB y SC. Otros investigadores ${ }^{(26-28)}$ han reportado que becerras $B$. taurus indicus requieren mayor peso y edad para iniciar la $\mathrm{PB}$ que becerras B. taurus taurus. Además, se ha descrito ${ }^{(29)}$ que en vaquillas Nelore una buena alimentación posdestete es un método efectivo para anticipar la PB. Entre las razas B. taurus indicus, la Nelore, que es la que aparentemente tiene menos colgajos y pliegues de piel y, por ende, menor superficie corporal, ha tenido mayor popularidad en Brasil, aparentemente por su mejor desempeño productivo, particularmente, mayor precocidad para iniciar la $\mathrm{PB}^{(29)}$ y 
menor edad al primer parto ${ }^{(30,31)}$; sin embargo, no se encontraron estudios que relacionen específicamente dichas características reproductivas con la SC de los animales.

La importancia de la relación entre superficie y masa corporal en el costo energético de mantenimiento de los animales ha sido reconocida y considerada desde hace más de un siglo; de hecho, entre los estudios iniciales sobre metabolismo basal, se discutió que la SC era tan importante o más importante que la masa corporal ${ }^{(32)}$. En estudios realizados con roedores, caninos, bovinos y humanos, se observó que la SC es una variable que permite la predicción más precisa de la tasa metabólica ${ }^{(33)}$. Por ello, se consideró como la variable que permitía, con mayor precisión, la comparación entre especies animales de diferentes tamaños en estudios cuantitativos de metabolismo ${ }^{(34)}$, lo que promovió el desarrollo de instrumentos para su medición ${ }^{(35)}$. No se encontraron trabajos específicamente diseñados para asociar la SC de los bovinos con algunas características productivas; en estudios previos ${ }^{(36,37)}$, se midió la SC de vacas lecheras y su relación con el peso corporal, para el uso de una fórmula basada en la "Ley de Kleiber" en estudios de bioenergética.

Se ha estimado que a mayor SC aumenta la pérdida de calor corporal por radiación, convección y evaporación $^{(38)}$, condiciones que implican un costo energético. Se ha evaluado que desde el nacimiento a medida que la masa corporal del animal aumenta con el crecimiento, su proporción relativa a la superficie corporal va también aumentando, y la disipación relativa de energía térmica corporal se reduce, permitiendo paulatinamente más energía disponible para los procesos fisiológicos y el almacenamiento de excedentes. Entre esos procesos fisiológicos no fundamentales para sostener la vida del individuo se encuentra la reproducción, que se vuelve factible una vez que la energía disponible en el organismo garantiza los procesos indispensables para la vida y otros prioritarios como la locomoción. Ello requiere un balance entre la energía que se consume y procesa y la que se acumula en tejidos, para después transformarse en trabajo o disiparse al ambiente como energía calórica; en esta última función, la piel y la respiración juegan un papel central en los bovinos ${ }^{(19)}$.

En el caso del ganado B. taurus indicus su mayor adaptación a climas cálidos, como los tropicales, se debe a su capacidad superior de regulación de la temperatura corporal durante condiciones de estrés por calor, derivada de una tasa metabólica más baja y mayor capacidad de disipación de calor a través de la piel $^{(39)}$. Conviene recordar que los animales que tienen mayor proporción de genes Cebú muestran menor tamaño de sus órganos torácicos y abdominales, como peso del rumen y longitud de los intestinos, que los animales $B$. taurus taurus $^{(40,41)}$. Se ha observado que la tasa metabólica basal en $B$. taurus indicus x B. taurus taurus es menor que en $B$. taurus taurus. En un estudio, la tasa de calor producido por unidad de superficie corporal de vacas no lactantes y en ayuno fue $57 \mathrm{MCal} / \mathrm{m}^{2}$ para Red Sindhi $\mathrm{x}$ Holstein y $100 \mathrm{MCal} / \mathrm{m}^{2}$ para Holstein ${ }^{(42)}$. 
Desde un punto de vista práctico, la mayor superficie de piel en Cebú por un lado le confiere ventajas (disipación de calor y resistencia al estrés térmico), pero aparentemente se asocia con una menor eficiencia en la utilización de la energía del alimento ${ }^{(38)}$, velocidad de crecimiento $^{(39)}$ y acumulación de energía en forma de grasa corporal ${ }^{(43,44)}$, que es un factor importante para la PB, como se reitera en los resultados de este trabajo, donde a pesar del uso de zilpaterol, que promovió un crecimiento más rápido, la pubertad se alcanzó a mayor edad, con mayor peso y musculatura en las vaquillas tratadas, pero con GD similar entre controles y tratados.

La suplementación con el $\beta$-agonista ZIL hizo que aumentara la EPB, y que las vaquillas control fueran más jóvenes a la PB. La administración de ZIL modifica la repartición de los nutrimentos, por lo que los animales crecen más rápido y ganan más peso, pero esta ganancia es más magra ${ }^{(45,46)}$. Para el inicio de la PB, no solo es importante la ganancia absoluta de peso de las vaquillas, es también importante la composición de la masa corporal ${ }^{(47-49)}$, ya que probablemente cambios sutiles o agudos en el estado metabólico inicien los eventos fisiológicos que conducen a la pubertad ${ }^{(5,50)}$. De ahí que, aunque las tratadas con ZIL llegaron con más peso y EPB, no fueron diferentes a las del grupo testigo en GD, a pesar de que estas últimas eran más livianas y con menor MD, lo que fue uno de los supuestos del estudio sobre el efecto de los $\beta$-agonistas en la repartición de los nutrimentos, ocasionando disminución de la lipogénesis y aumento de la acreción muscular, como han observado diversos autores, que han reportado que la suplementación con ZIL aumentó la MD y disminuyó el GD en vacas ${ }^{(51)}$, vaquillas $^{(52)}$ y novillos ${ }^{(53)}$.

La información disponible indica que se requiere un mínimo de grasa corporal para detonar procesos reproductivos como la $\mathrm{PB}$ en vaquillas ${ }^{(9)}$ y un mínimo de $\mathrm{CC}^{(54)}$. En un estudio ${ }^{(55)}$ realizado en vaquillas Nelore, se encontraron altas correlaciones (de 0.82 a 0.93 ) entre la CC y el GD, y se afirmó que con la CC se puede predecir el GD en ganado $B$. taurus indicus en las diferentes etapas del ciclo de producción. En un estudio con vacas B. taurus taurus $^{(56)}$, se observó que un incremento de la CC tendía a estar acompañado por un aumento de tamaño de los adipocitos en el tejido adiposo subcutáneo. La información presentada indica que los animales $B$. taurus indicus necesitan mayor acumulación de grasa que los B. taurus taurus para iniciar la PB, pudiéndose lograr esto mediante dietas con más energía. En el trabajo motivo de esta comunicación, esas relaciones fueron modificadas por el uso del $\beta$-agonista ZIL en ambas razas, y las vaquillas tratadas fueron más musculosas y tuvieron mayor $\mathrm{CC}$ y más MD a la $\mathrm{PB}$, como era de esperarse ${ }^{(5)}$, pero no hubo diferencia con las del grupo testigo en GD a la PB.

Es manifiesto que en BHM, por encima de los señalizadores internos relacionados con el peso y composición corporal, prevaleció un efecto de estacionalidad asociado con los cambios de luz; por ello, se observó efecto de RZ sobre EPB y diferencias entre razas en los 
meses de presentación de la PB. El efecto de estacionalidad se manifestó en que las vaquillas SE iniciaron la PB de manera homogénea a través del año, pero $73.8 \%$ de las vaquillas BHM iniciaron la PB en los meses en que las horas luz iban en aumento y sólo $26.2 \%$ lo hizo cuando las horas luz decrecían $(P<0.05)$. De hecho, el efecto de estacionalidad se manifiesta sobre otras variables, como se muestra en el Cuadro 2, donde se mantuvieron las diferencias en variables que se consideraron como críticas para el inicio de la PB, como es el caso de PM/SC, GD y LEP, en animales nacidos en diferentes estaciones. La observación del efecto de estacionalidad sobre la PB en BHM coincide con lo descrito por otros autores ${ }^{(3)}$, que observaron que vaquillas BHM sólo iniciaron la pubertad en un periodo comprendido de febrero a mayo (días en los que las horas luz van en aumento) a diferencia de vaquillas Suizo Pardo que iniciaron la pubertad a lo largo del año. Dicho efecto lo atribuyeron a la susceptibilidad de las becerras BHM a los efectos ambientales que determinan las estaciones del año (estacionalidad). Estas observaciones coinciden con resultados de otros estudios con hembras Cebú ${ }^{(11,12,57)}$, donde se observó una tendencia estacional en la actividad reproductiva posparto de las vacas, aún en condiciones de alimentación controlada. El fotoperiodo parece influir en el inicio de la pubertad. En un experimento con vaquillas lecheras, la iluminación suplementaria (16 h de luz/día) durante el invierno mejoró las tasas de crecimiento y redujo la edad a la pubertad ${ }^{(58)}$. De igual forma, con vaquillas B. taurus taurus, la iluminación suplementaria ( 18 h de luz/día) después de las 22 o 24 semanas de edad redujo la edad a la pubertad en las vaquillas nacidas de febrero a julio. Estos efectos del fotoperiodo se acompañaron de cambios en el desarrollo ovárico ${ }^{(59)}$. El mismo autor ${ }^{(60)}$ señala que vaquillas con propensión genética a alcanzar la pubertad a edades tempranas, pueden verse afectadas por la estación de nacimiento de manera diferente a aquellas que alcanzan la pubertad a edades más avanzadas.

En comparación con las vaquillas SE, las vaquillas BHM fueron más susceptibles a señales ambientales por el cambio de horas luz, lo cual probablemente desencadenó los procesos neuroendocrinos asociados con el inicio de la PB, situación que debe considerarse en la planificación de los programas de manejo reproductivo de bovinos en hatos con una composición racial diversa. El efecto de la estación de nacimiento influyó en que las vaquillas nacidas en otoño tuvieron mayor GD y niveles más elevados de LEP a la PB que las nacidas en primavera, pero sin haber diferencias $(P>0.05)$ en EPB entre estaciones de nacimiento en las condiciones de este estudio.

Se observó interacción de RZ x EN sobre CC a la pubertad, donde las vaquillas SE nacidas en primavera mostraron menor CC que las vaquillas SE nacidas en otoño y que las BHM nacidas en primavera. Con base en promedios generales (no mostrados aquí, pueden consultarse en un estudio previo ${ }^{(61)}$ ), aparentemente, las vaquillas de menor EPB (BHM nacidas en primavera y SE nacidas en otoño) llegaron con mayor CC (Figura 1), aunque las diferencias en esta variable fueron mínimas. A este respecto, diversos autores han sugerido 
que hay un fenómeno de compensación de edad con peso para el establecimiento de la $\mathrm{PB}^{(49,62,63)}$.

Se ha informado que en vacas Bos taurus taurus ${ }^{(56)}$ hay una correlación positiva de la leptina con peso corporal y CC; además, un incremento de la CC tendía a estar acompañado por un aumento de tamaño de los adipocitos en el tejido adiposo subcutáneo. En el presente estudio, la LEP a la PB fue diferente entre razas; las vaquillas BHM mostraron mayor LEP que las SE. Esta diferencia de LEP entre razas puede deberse a que las BHM fueron las que también presentaron mayor edad y cantidad de GD a la PB, ya que los niveles circulantes de leptina

están directamente asociados con la adiposidad corporal ${ }^{(64,65)}$. Hasta el momento no hay evidencia de una transición abrupta en las concentraciones plasmáticas prepuberales de leptina a la pubertad o de que las concentraciones circulantes puedan ser un desencadenante crítico para la PB en vaquillas de crecimiento rápido, pero aparentemente se requiere un mínimo de leptina circulante para la $\mathrm{PB}$ en vaquillas con tasas de crecimiento normal o restringido $^{(66)}$.

\section{Conclusiones e implicaciones}

Se concluye que la masa corporal como proporción de la SC parece tener un papel importante en el inicio de la PB de vaquillas y que se requiere un mínimo de masa de tejidos metabólicamente activos por unidad de SC y un mínimo de acumulación de grasa para que se detone el inicio de la PB, independientemente de la raza de que se trate. El establecimiento de la pubertad es un fenómeno complejo, que depende de la interacción entre variables y señalizadores internos de los animales y de otros factores de su entorno, como los cambios de las horas luz, que afectan a las vaquillas Cebú. Las observaciones derivadas de este trabajo permiten especular que la pubertad tardía de las vaquillas BHM puede estar asociada con la mayor SC en comparación con las vaquillas SE y ratifica el efecto de los cambios de horas luz en fenómenos reproductivos en hembras Cebú, aun cuando se elimina el efecto indirecto de estacionalidad a través de la alimentación controlada. Este trabajo documenta por primera ocasión la relación de la masa corporal, como proporción de la superficie corporal, con el establecimiento de la pubertad en bovinos.

\section{Conflicto de intereses}

Los autores declaran que no existe ningún conflicto de intereses en la presentación de este trabajo. 


\section{Literatura citada:}

1. Day ML, Nogueira GP. Management of age at puberty in beef heifers to optimize efficiency of beef production. Anim Front 2013;3:6-11.

2. Manthey AK, Anderson JL, Perry GA, Keisler DH. Feeding distillers dried grains in replacement of forage in limit-fed dairy heifer rations: Effects on metabolic profile and onset of puberty. J Dairy Sci 2017;100:1-12.

3. Calderón RRC, Villa-Godoy A, Lagunes LJ. Determinación ultrasonográfica de la primera ovulación: asociación con la primera ovulación de ciclos estrales regulares en vaquillas Cebú y Suizo Pardo mantenidas en el trópico. Téc Pecu Méx 1996;34(2):79-88.

4. Moriel P, Piccolo M, Lancaster PA, Lamb GC, Vendramini J, Arthington JD. Effects of post-weaning plane of nutrition and estrus synchronization on reproductive performance of influenced beef heifers. J Anim Sci 2017;95(4):3523-3531.

5. Hall JB, Staigmiller RB, Bellows RA, Short RE, Moseley WM, Bellows SE. Body composition and metabolic profiles associated with puberty in beef heifers. J Anim Sci 1995;73(11):3409-3420.

6. Gonzalez-Padilla E, Wiltbank JN, Niswender GD. Puberty in beef heifers. I. The interrelationship between pituitary, hypothalamic and ovarian hormones. J Anim Sci 1975;40(6):1091-1104.

7. Foote AP, Tait RG, Keisler DH, Hales KE, Freetly HC. Leptin concentrations in finishing beef steers and heifers and their association with dry matter intake, average daily gain, feed efficiency, and body composition. Domest Anim Endocrinol 2016;55:136-141.

8. Garcia MR, Amstalden M, Williams SW, Stanko RL, Morrison CD, Keisler DH et al. Serum leptin and its adipose gene expression during pubertal development, the estrous cycle, and different seasons in cattle. J Anim Sci 2002;80(8):2158-2167.

9. Pardo AM, Villareal EL, Papaleo MJ, Melucci OG, Santamaría S, Ferrario J et al. Sexual precocity and productivity of beef cattle female under grazing conditions. Anim Prod Sci 2018;59:757-766.

10. Johnston DJ, Barwick SA, Corbet NJ, Fordyce G, Holroyd RG, Williams PJ et al. Genetics of heifer puberty in two tropical beef genotypes in northern Australia and associations with heifer- and steer-production traits. Anim Prod Sci 2009;49:399-412.

11. Lozano DRR, Asprón PMA, González-Padilla E, Vásquez PCG. Estacionalidad reproductiva en vacas Bos indicus en el trópico mexicano. Téc Pecu Méx 1987;2(25):192-205. 
12. Villagómez AME, Castillo RH, Villa-Godoy A, Román PH, Vázquez PC. Influencia estacional sobre el ciclo estral y el estro en hembras cebú mantenidas en clima tropical. Téc Pecu Méx 2000;38(2):89-103.

13. Evans ACO, Adams GP, Rawlings NC. Endocrine and ovarian follicular changes leading up to the first ovulation in prepuberal heifers. J Reprod Fertil 1994;100:187-194.

14. Richards MW, Spitzer JC, Warner MB. Effect of varying levels of postpartum nutrition and body condition at calving on subsequent reproductive performance in beef cattle. $\mathbf{J}$ Anim Sci 1986;62:300-306.

15. SPSS Inc. PASW Statistics for Windows, Version 18.0. Chicago: SPSS Inc. 2009.

16. Perkins TL, Green RD, Hamlin KE. Evaluation of ultrasonic estimates of carcass fat thickness and longissimus muscle area in beef cattle. J Anim Sci 1992;70:1002-1010.

17. Delavaud C, Bocquier F, Chilliard Y, Keisler DH, Gertler A, Kann G. Plasma leptin determination in ruminants: Effect of nutritional status and body fatness on plasma leptin concentration assessed by a specific RIA in sheep. J Endocrinol 2000;165:519-526.

18. SAS. Statistical Analysis System Institute Inc, Cary, NC, USA. 2002.

19. Foster DL, Hileman SM. Puberty in sheep. Knobil and Neill's Physiology of Reproduction. Fourth ed. 2015;1441-1485.

20. Alberti P, Panea B, Sanudo C, Olleta JL, Rpoll G, Ertbjerg P et al. Live weight, body size and carcass characteristics of young bulls of fifteen European breeds. Livest Sci 2008;114:19-30.

21. Clarke AM, Drennan MJ, McGee M, Kenedy DA, Evans RD, Berry DP. Intake, live animal scores/measurements and carcass composition and value of late-maturing beef and dairy breeds. Livest Sci 2009;126:57-68.

22. Thrift FA, Sanders JO, Brown AH, Herring AD, Riley DG, DeRouen SM. Preweaning, postweaning, and carcass trait comparisons for progeny sired by subtropically adapted beef sire breeds at various US locations. The Prof Anim Scientist 2010;26(5):451-473.

23. McGee M, Keane MG, Neilan R, Moloney AP, Caffrey PJ. Production and carcass traits of high dairy genetic merit Holstein, standard dairy genetic merit Friesian and Charolais x Holstein-Friesian male cattle. Irish J Agr Food Res 2005;44:215-231.

24. Black JL. Animal growth and its regulation. J Anim Sci 1988;66:1-22.

25. Nogueira GP. Puberty in South American Bos indicus (Zebu) cattle. Anim Reprod Sci 2004;82-83:361-372. 
26. Reynolds WL, de Rouen TM, High Jr JW. The age and weight at puberty of Angus, Brahman and zebu cross heifers [abstract]. J Anim Sci 1963;22:243.

27. Calderón RRC. Cambios dinámicos de las estructuras ováricas y su relación con la progesterona sérica en becerras peripúberes Bos taurus y Bos indicus, mantenidas en clima tropical [tesis maestría]. México, DF: Universidad Nacional Autónoma de México; 1994.

28. Pereira GR, Barcellos JOJ, Sessim AG, Tarouco JU, Feijó FD, Neto JB et al. Relationship of post-weaning growth and age at puberty in crossbred beef heifers. Rev Bras Zootec 2017;46(5):413-420.

29. Nepomuceno DD, Pires AV, Ferraz MVC, Biehl MV, Gonçalves JRS, Moreira EM et al. Effect of pre-partum dam supplementation, creep-feeding and post-weaning feedlot on age at puberty in Nellore heifers. Livest Sci 2017;195:58-62.

30. Eler JP, Silva JVA, Ferraz JBS, Dias F, Oliveira NH, Evans JL et al. Genetic evaluation of the probability of pregnancy at 14 months for Nellore heifers. J Anim Sci 2002;80:951-954.

31. Nogueira GP, de Lucia RFS, Pereira FV, Cirilo PD. Precocious fertility in Nelore heifers [abstract]. Biol Reprod 2003;68(Suppl 1):382.

32. Richet C. La Chaleur Animale. Paris, 1889.

33. Kleiber M. Body size and metabolism. Hilgardia 1932;6:315-353.

34. Kleiber M. Body size and metabolic rate. Physiol Rev 1947;4(27): 511-541.

35. Mitchell HH. The effect of the amount of feed consumed by cattle on the utilization of its energy content. J Agric Res 1932;3(45):163-191.

36. Hogan AG, Skouby CI. Determination of the surface area of cattle and swine. J Agric Res 1923;19(25):419-430.

37. Elting ECA. Formula for estimating surface area of dairy cattle. J Agric Res 1926;33(3):269-280.

38. Berman A. Effects of body surface area estimates on predicted energy requirements and heat stress. J Dairy Sci 2003;86(11):3605-3610.

39. Hansen P. Physiological and cellular adaptations of zebu cattle to thermal stress. Anim Reprod Sci 2004;82-83:349-360. 
40. Swett WW, Matthews CA, McDowell RE. 1961. Sindhi-Jersey and Sindhi-Holstein crosses: their external form and internal anatomy compared with those of purebred Jerseys and Holsteins. Tech Bull 1961. USDA 1236.

41. McDowell RE, Wilk JC, Talbott CW. Economic viability of crosses of Bos taurus and Bos indicus for dairying in warm climates. J Dairy Sci 1996;79(7):1292-1303.

42. Johnston JE, Hamblin FB, Schrader GT. Factors concerned in the comparative heat tolerance of Jersey, Holstein, and Red Sindhi-Holstein (F1) cattle. J Anim Sci 1958; $17: 473-479$.

43. Bucholtz DC, Manning J, Herbosa CG, Schillo KK, Foster DL. The energetics of LH secretion: a temporally-focused view of sexual maturation [abstract]. In: Annual Meeting of the Society for Neuroscience, Washington, D.C. 1993;23:349.

44. Frisch RE. Body weight, body fat and ovulation. Trends Endocrinol Metab 1991;2:191197.

45. Moloney AP, Beermann DH. Mechanisms by wich $\beta$-adrenergic agonists alter growth and body composition in ruminants. In: Enne G, et al, editors Residues of veterinary drugs and mycotoxins in animal products. Pers, Wageningen 1996;124-136.

46. Cônsolo NRB, Rodriguez FD, Goulart RS, Frasseto MO, Ferrari VB, Silva LFP. Zilpaterol hydrochloride improves feed efficiency and changes body composition in nonimplanted Nellore heifers. J Anim Sci 2015;93(10):4948-4955.

47. Maciel MN, Zieba DA, Amstalden M, Keisler DH, Neves J, Williams GL. Recombinant leptin prevents fasting-mediated reductions in pulsatile $\mathrm{LH}$ release and stimulates $\mathrm{GH}$ secretion in peripubertal heifers [abstract]. Proc Mid West Sect Am Soc Anim Sci $2003 ; 66: 263$.

48. Arije GF, Wiltbank JN. Age and weight at puberty in Hereford heifers. J Anim Sci 1971;33:401-406.

49. González-Padilla E, Ruíz DR, Wiltbank JN. Inducción y sincronización del estro en vaquillas prepúberes mediante la administración de estrógenos y un progestágeno. Téc Pecu Méx 1975;(1):17-23.

50. Steiner RA, Cameron JL, McNeill TH, Clifton DK, Bremner WJ. Metabolic signals for the onset of puberty. In: Norman RL, editor. Neuroendocrine aspects of reproduction. Academic Press, New York 1983.

51. Lowe BK, Mckeith RO, Segers JR, Safko JA, Froetschel MA, Stewart Jr RL et al. The effects of zilpaterol hydrochloride supplementation on market dairy cow performance, carcass characteristics, and cutability. Prof Anim Scient 2012;28(2):150-157. 
52. Rathmann RJ, Bernhard BC, Swingle RS, Lawrence TE, Nichols WT, Yates DA. Effects of zilpaterol hydrochloride and days on the finishing diet on feedlot performance, carcass characteristics, and tenderness in beef heifers. J Anim Sci 2012;90:3301-3311.

53. Kononoff PJ, Defoor PJ, Engler MJ, Swingle RS, James ST, Deobald HM et al. Impact of a leptin single nucleotide polymorphism and zilpaterol hydrochloride on growth and carcass characteristics in finishing steers. J Anim Sci 2013;91(10):5011-5017.

54. Perry GA. Physiology and endocrinology symposium: harnessing basic knowledge of factors controlling puberty to improve synchronization of estrus and fertility in heifers. J Anim Sci 2012;90(4):1172-1182.

55. Ayres H, Ferreira RM, de Souza Torres-Júnior JR, Demétrio CGB, de Lima CG, Baruselli PS. Validation of body condition score as a predictor of subcutaneous fat in Nelore (Bos indicus) cows. Livest Sci 2009;123(2-3):175-179.

56. Locher L, Häussler S, Laubenthal L, Singh SP, Winkler J, Kinoshita A et al. Effect of increasing body condition on key regulators of fat metabolism in subcutaneous adipose tissue depot and circulation of nonlactating dairy cows. J Dairy Sci 2015;98(2):10571068.

57. Plasse D, Warnick AC, Koger M. Reproductive behavior of Bos indicus females in a subtropical environment. I. Puberty and ovulation frequency in Brahman and Brahman x British heifers. J Anim Sci 1968;27:94-100.

58. Peters RR, Chapin LT, Leining KB, Tucker HA. Supplemental lighting stimulates growth and lactation in cattle. Science 1976;199:911-912.

59. Hansen PJ, Kamwanja LA, Hauser ER. Photoperiod influences age at puberty of heifers. J Anim Sci 1983;57:985-992.

60. Hansen PJ. Seasonal modulation of puberty and the postpartum anestrus in cattle: A review. Livest Prod Sci 1985;12:309-327.

61. Hernández LC. Interacciones entre estacionalidad, características corporales y leptina en el establecimiento de la pubertad en vaquillas Bos taurus taurus y Bos taurus indicus [tesis maestría]. México, CDMX: Universidad Nacional Autónoma de México; 2018.

62. Greer RC, Whitman RW, Staigmiller RB, Anderson DC. Estimating the impact of management decisions on the occurrence of puberty in beef heifers. J Anim Sci 1983;56(1):30-39.

63. Ferreira VCP, Penna VM, Bergmann JAG, Torres RA. Interação genótipo-ambiente em algumas características produtivas de gado de corte no Brasil. Arq Bras Med Vet Zootec 2001;53:385-392. 
64. Ehrhardt R. Development of a specific radioimmunoassay to measure physiological changes of circulating leptin in cattle and sheep. J Endocrinology 2000;166(3):519-528.

65. Geary TW, McFadin EL, MacNeil MD, Grings EE, Short RE, Keisler DH. Leptin as a predictor of carcass composition in beef cattle. J Anim Sci 2003;81(1):1-8.

66. Chelikani PK, Ambrose DJ, Keisler DH, Kennelly JJ. Effects of dietary energy and protein density on plasma concentrations of leptin and metabolic hormones in dairy heifers. J Dairy Sci 2009;92(4):1430-1441. 